\title{
WEED COMMUNITIES IN POTATO (Solanum tuberosum L.)CROPS OF THE MAZOWIECKI LANDSCAPE PARK
}

\author{
Maria Ługowska, Teresa Skrajna \\ Department of Agricultural Ecology, Siedlce University of Natural Sciences and Humanities \\ B. Prusa 14, 08-110 Siedlce, Poland \\ e-mail: ekorol@uph.edu.pl
}

Received: 22.05.2012

\begin{abstract}
This paper presents a description of segetal communities in potato crops cultivated in the Masovian Landscape Park. The communities were analysed based on 64 phytosociological relevés made at 45 localities. 4 associations were found in the study area, that is, Digitarietum ischaemi, Echinochloo-Setarietum, Galinsogo-Setarietum, and Lamio-Veronicetum politae. Phytocenoses representing the associations Digitarietum ischaemi and Echinochloo-Setarietum were most varied floristically. Lower syntaxonomical units were determined within these associations, that is, subassociations and variants. These phytocenoses were frequently found; they occurred in various habitats characterized by different trophic and moisture conditions. Patches of the association Lamio-Veronicetum polita were rare and they occurred only on fertile soils and on small areas.
\end{abstract}

Key words: root crops, weed communities, biodiversity of weeds, Mazowiecki Landscape Park

\section{IINTRODUCTION}

The area under potato has fallen considerably over the last decade from 1250.4 (in 2000) to 388.3 th (in 2010) (GUS, 2011). This has resulted from changes in the cropping structure, increased fallow area, an unfavourable economic situation, and in the case of land in the vicinity of urban areas, the allocation of land for building development. As far as the Masovian Landscape Park is concerned, there is an additional element, that is, the land use structure: $70 \%$ of the area is covered by forest, whereas $11.3 \%$ is ploughed land. All the aforementioned factors have influenced potato cultivation in the Masovian Landscape Park causing its continuous decline. The areas cropped to potato are small and yields harvested are mainly used to meet the needs of households.

This paper presents the next study on phytocenoses established in crop plants in this area ( $\mathrm{S} \mathrm{k} \mathrm{r} \mathrm{a} \mathrm{j} \mathrm{n} \mathrm{a}$ et al. 2009; S kra jn a and $€$ u g o w sk a, 2010). The objective of the study was to analyse communities in potato crops against the background of various habitats under conditions of extensive farming in the Masovian Landscape Park area.

\section{RESEARCH METHODS AND AREA}

Observations of segetal communities in the Masovian Landscape Park area were conducted in the years 2003-2008. 64 phytosociological relevés were made by the Braun-Blanquet method ( $\mathrm{P}$ a w $\mathrm{fow} \mathrm{s} \mathrm{ki}$, 1972) in 45 localities (Fig. 1) within the Park and its buffer zone. During the field study, agricultural soil maps at a scale of 1:5000 were used to select sites for examination and to determine soil conditions. The factual material collected was analysed phytosociologically based on the rules suggested by M t u s z kiewicz (2007). Species nomenclature followed Mirek et al. (2002). 


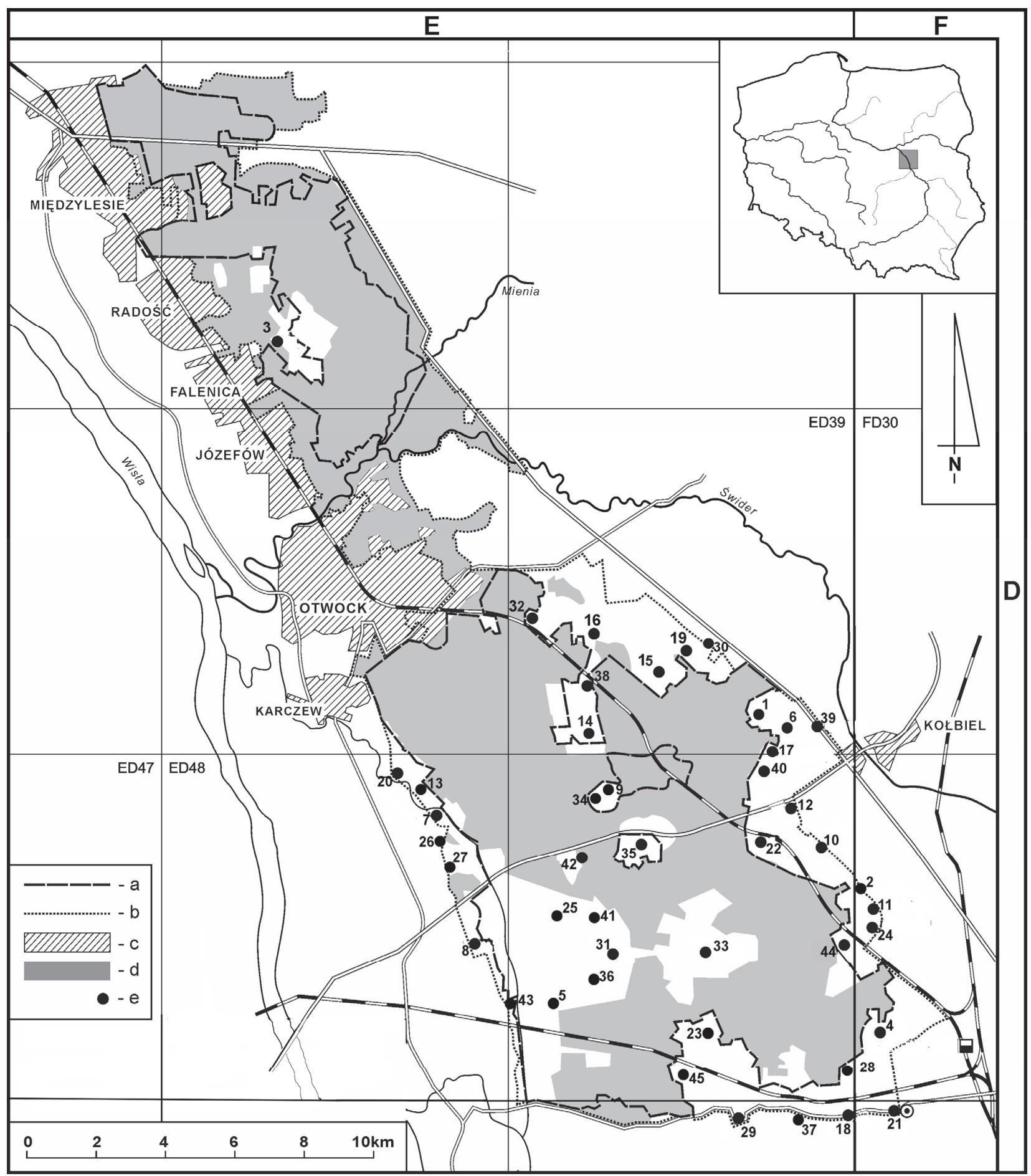

Figure 1. Study area

$\mathrm{a}$ - borders of the Landscape Park; $\mathrm{b}$ - borders of the buffer zone; $\mathrm{c}$ - built-up areas; $\mathrm{d}$ - forest complexes; $\mathrm{e}$ - localities, study sites.

1 -Anielinek, 2 - Antoninek, 3 - Aleksandrówka, 4 - Augustówka, 5 - Bąki, 6 - Bocian, 7 - Brzezinka, 8 - Całowanie, 9 - Celestynów, 10 - Chrosna, 11 - Chrząszczówka, 12 - Człekówka, 13 - Dąbrowa, 14 - Dąbrówka, 15 - Dyzin, 16 - Glina, 17 - Gózd, 18 - Grabianka, 19 - Jatne, 20 Janów, 21 - Jaźwiny, 22 - Karpiska, 23 - Kąciki, 24 - Kąty, 25 - Kominki, 26 - Kozłówka, 27 - Łukowiec, 28 - Ocznia, 29 - Osieck, 30 - Ostrowik, 31 - Podbiel, 32 - Pogorzel, 33 - Ponurzyca, 34 - Radzyń, 35 - Regut, 36 - Rosłańce, 37 - Rudnik, 38 - Stara Wieś, 39 - Stara Wieś II, 40 - Skorupy, 41 - Szatany, 42 - Tabor, 43 - Warszówka, 44 - Zabieżki, 45 - Zawada. 


\section{RESULTS}

Ten different types of phytocenoses, represented by subassociations and variants of 4 associations, were recognised in the Masovian Landscape Park.

Systematics of distinguished root crops communities

Class: Stellarietea mediae Tx.,

Lohm. et Prst. 1950

Order: Polygono-Chenopodietalia

(R.Tx. et Lohm. 1950) J. Tx. 1961

Alliance: Panico-Setarion Siss. 1946

1. Association: Digitarietum ischaemi R. Tx. et Prsg (1942) 1950

a. typical variant

b. variant with a share of ruderal species

c. variant with a share of wet species

2. Association: Echinochloo-Setarietum Krusem. et Vlieg. (1939) 1940

Subassociation:

Echinochloo-Setarietum sperguletosum

Subassociation:

Echinochloo-Setarietum typicum

a. typical variant

b. variant with a share of wet species

c. variant with Amaranthus retroflexus

d. variant with Veronica agrestis

Alliance: Polygono-Chenopodion Siss. 1946

1. Association: Galinsogo-Setarietum (R. Tx et Beck. 1942) R. Tx. 1950

2. Association: Lamio-Veronicetum politae Kornaś 1950

\section{Digitarietum ischaemi $\mathrm{R}$.}

Tx. Et Prsg (1942) 1950

The association, of Sub-Atlantic character, occurred both in the Park and its buffer zone.

It was described on the basis of 17 phytosociological relevés. Altogether, there were recorded 71 species in all the examined patches of land (Table 1). Due to the great floristic diversity within this association, apart from the typical form, there was found a form characterised by the presence of ruderal and wet species. The appearance of the community was dominated by the typical species Digitaria ischaemum, with its percentage exceeding $60 \%$ in some patches.

Typical patches of this community developed on dry and poor soils. 42 taxa were found that were the poorest phytocenoses in the potato fields. The nature of the analysed phytocenoses was influenced by the group of acidophilous species representing the association Panico-Setarion and which included Rumex acetosella, Spergula arvensis, Scleranthus annuus, Setaria pumila and Raphanus raphanistrum.

Patches of this variant including ruderal species formed in small areas cropped to potato located adjacent to fallow land. This variant was distinguished because the community was characterised by a considerable share of ruderal species compared with other agrophytocenoses. Frequent components of the flora of this community were as follows: Artemisia vulgaris, Erigeron acer, Lactuca serriola, Tanacetum vulgare, Malva neglecta, Sisymbrium officinale.

On light loamy sands, representing different soil types belonging to the cereal-fodder poor soil quality complex, phytocenoses formed that included moisture-loving species. The variant with these species was distinguished based on 5 plant patches. They were slightly richer phytocenoses than the previous ones as 44 weed species were found. The nature of this community was defined, apart from the typical species, by moisture-loving species with shallow roots, the most numerous and most frequent species being Spergularia rubra, Gnaphalium uliginosum, Juncus bufonius, Sagina procumbens, and Plantago intermedia.

Echinochloo-Setarietum Krusem.

et Vlieg. (1939) 1940

The association Echinochloo-Setarietum was characterised on the basis of 26 phytosociological relevés which comprised 114 weed species (Table 2). These phytocenoses were most frequently found in the examined fields. The diversity of habitats where the phytocenoses of this association developed influenced the heterogeneity of individual patches. Within the association, 2 subassociations and 3 variants were found. The whole association was influenced by the mass presence of its typical species, Echinochloa crus-galli, which reached a very high cover index ( $\mathrm{D}=2567)$. In turn, Raphanus raphanistrum was most frequently found in Echinochloo-Setarietum typicum, in the remaining communities being found in parts of the patches only. Moreover, the common species in the association were Setaria pumila, Matricaria maritima subsp. indora, Chenopodium album, Stellaria media, Viola arvensis, Polygonum aviculare, and Polygonum lapathifolium subsp. lapathifolium.

Relevés 1 to 5 revealed communities of the most acidophilous character which were included in Echinochloo-Setarietum sperguletosum. Patches of this subassociation developed on different types of soils representing the good and poor rye soil quality complex. It was the floristically poorest community in the association Echinochloo-Setarietum. Only 39 weed species were found in it. The nature of the association was defined by 3 acidophilous species: 
Spergula arvensis, Rumem acetosella, Scleranthus annuus.

Typical patches of the association were found on leached brown soils belonging to the very good and good rye soil quality complex. The community comprised 54 weed species. Apart from the species typical of this association, Matricaria maritima subsp. indora was a regular component of these phytocenoses, whereas Chenopopodium album was the most frequent component of the syntaxa of this association.

The association Echinochloo-Setarietum, with a high share of wet species, was found on light and strong loamy sands representing degraded chernozems and alluvial soils. These were the richest phytocenoses in this association, represented by 73 weed species. The following were the most frequent and numerous species: Plantago intermedia, Gnaphalium uliginosum, Mentha arvensis, Ranunculus repens, Polygonum hydropiper.

Some patches of the association Echinochloo-Setarietum were characterised by a substantial share of Amaranthus retroflexus, which made it possible to classify these phytocenoses as a variant including this species. The community developed on various soils and was floristically abundant, as 70 species were found in 5 relevés. Numerous Amaranthus retroflexus plants in the communities were accompanied by other species, such as Stellaria media, Polygonum lapathifolium subsp. lapathifolium, and Senecio vulgaris, which is indicative of high soil nitrogen content in these habitats. The analysed phytocenoses comprised a small number of wet species.

Communities representing Echinochloo-Setarietum with Veronica agrestis established on trophically rich and well-moistened fertile soils. The phytocenoses were characterised by a high share of species of the alliance Polygono-Chenopodion, of which Veronica persica, Lamium purpureum and Euphorbia helioscopia were regular components.

\section{Galinsogo-Setarietum}

(R. Tx et Beck. 1942) R. Tx. 1950

Patches of the association Galinsogo-Setarietum developed on leached brown soils which were well fertilised and in good tilth. These phytocenoses were most frequent in the Park's buffer zone, mainly in the proximity of buildings. The association was described based on 10 phytosociological relevés which were floristically rich communities. There were found 76 species in all the examined patches, the average number of species per relevé being 25 (Table 3 ). The regular components of the phytocenoses were species typical of the association, of which Galinsoga parviflora had a very high cover index. The analysed patches were characterised by a high share of the alliance Polygono-Chenopodion species, the most frequent being Lamium purpureum, Veronica persica, Euphorbia helioscopia and Matricaria maritima subsp. inodora. Moreover, the nature of the phytocenoses was also influenced by species belonging to higher syntaxonomic units which locally reached higher cover, that is, Chenopodium album, Stellaria media, Setaria pumila, Viola arvensis, and Anthemis arvensis.

\section{Lamio-Veronicetum politae}

Kornaś 1950

Patches of the association developed on fertile $\mathrm{CaCO}_{3}$-rich soils of the good wheat soil quality complex. The association is found both in the Park and in its buffer zone. The phytocenoses were floristically rich as 82 weed species were recorded in 10 patches, the average number of species per relevé being 26 (Table 4). Of the typical species, Lamium amplexicaule was the most common component of this association, Veronica agrestis was less frequent, whereas Veronica opaca and Veronica polita occurred only in some plant patches. There was found a considerable group of the Polygono-Chenopodion alliance species, of which the most numerous species that reached the highest cover were: Veronica persica, Lamium purpureum, Chenopodium polyspermum, Euphorbia helioscopia and Oxalis fontana. In turn, common species of higher syntaxonomic units in the association included: Chenopodium album, Stellaria media, Anagallis arvensis, Chaenorchinum minus, Echinochloa crus-galli, Polygonum lapathifolium subsp. pallidum, and Aethusa cynapium. 
Table 1

Digitarietum ischaemi R. Tx. et Prsg (1942) 1950

Subassociation

Variant

Relevé no. in table

Relevé no.in field

Date: month

year

Locality

Crop cover in \%

Weed cover in \%

Soil unit

Number of species per relevé

I. Ch.D. Digitarietum ischaem

$\begin{array}{lllllll}16 & 15 & 15 & 17 & 13 & 16 & 15\end{array}$

135157139193141154145

$\begin{array}{lllllll}8 & 8 & 8 & 9 & 8 & 8 & 8\end{array}$

$\begin{array}{lllllll}.06 & 06 & 06 & 06 & 06 & 06 & 06\end{array}$

$\begin{array}{lllllll}3 & 21 & 9 & 42 & 11 & 18 & 13\end{array}$

60 zn $\quad$ zn $\quad$ zn $\quad$ zn $\quad$ zn $\quad$ zn

$\begin{array}{lllllll}40 & 70 & 75 & 75 & 70 & 55 & 75\end{array}$ with ruderal species

$$
\begin{array}{lllllll}
1 & 2 & 3 & 4 & 5 & 6 & 7
\end{array}
$$

$\begin{array}{lllll}8 & 9 & 10 & 11 & 12\end{array}$

130197180149159

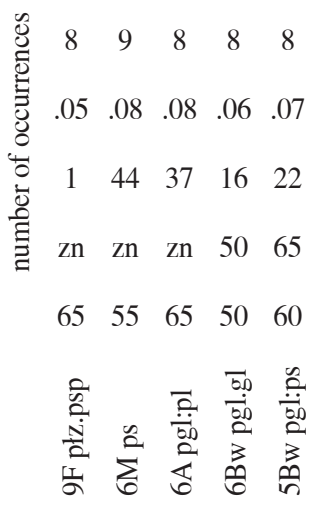

151148156136137

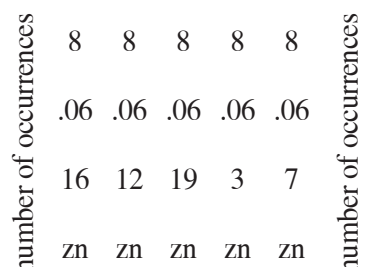

$\begin{array}{lllll}60 & 55 & 75 & 55 & 75\end{array}$

$\begin{array}{lllll}19 & 18 & 22 & 21 & 19\end{array}$

Digitaria ischaemum (Schreb.) Mühlenbg.

\section{D var. with ruderal species}

Artemisia vulgaris $\mathrm{L}$.

Solidago canadensis L.

Erigeron acris L.

Malva neglecta Wallr.

Lactuca serriola $\mathrm{L}$.

Tanacetum vulgare $\mathrm{L}$.

Sisymbrium officinale L.

\section{D. var. with of wet}

Polygonum hydropiper L.

Plantago intermedia Gilib.

Spergularia rubra $(\mathrm{L}$.

J. Presl \& C. Presl

Gnaphalium uliginosum L.

Juncus bufonius L.

Sagina procumbens L.

Phragmites australis (Cav.)

Trin. et Steud.

Bidens tripartita $\mathrm{L}$. $\begin{array}{llllllllllllllllllllll}1 & 4 & 4 & 3 & 2 & 3 & 1 & 7 & 2 & 2 & 3 & 2 & 3 & 5 & 2 & 2 & 1 & 2 & 2 & 5 & \mathrm{~V} & 2529\end{array}$

\begin{tabular}{|c|c|c|c|c|c|c|c|c|c|c|c|}
\hline & + & 2 & 1 & + & 1 & 5 & + & & & 1 & III \\
\hline & + & & 1 & 1 & + & 4 & & & & & II \\
\hline & & & + & 1 & 1 & 3 & & + & & 1 & II \\
\hline & + & & 1 & & + & 3 & & & + & 1 & II \\
\hline & + & + & & + & + & 4 & + & & & 1 & II \\
\hline & + & + & & + & + & 4 & & & & & II \\
\hline & & + & + & & + & 3 & & & & & II \\
\hline
\end{tabular}

$+$

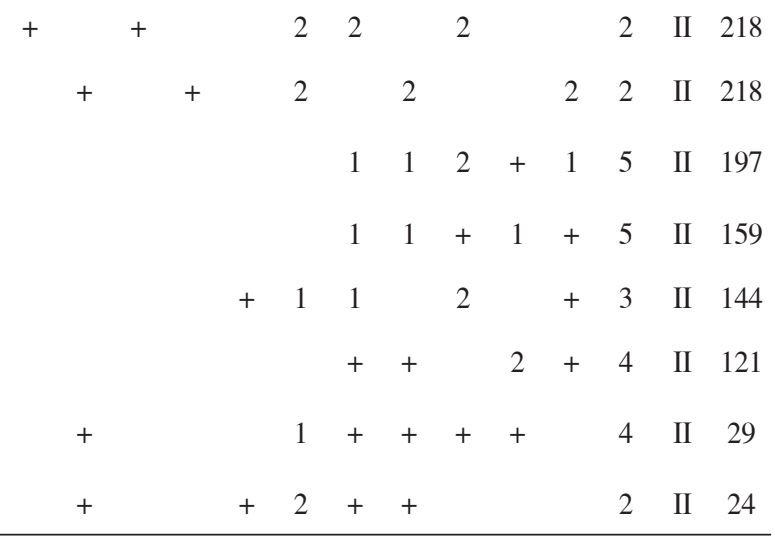


IV. Ch.D. Panico-Setarion,

Polygono-Chenopodietalia

Scleranthus annuus L.

Setaria pumila (Poir.)

Roem. \& Schult.

Rumex acetosella $\mathrm{L}$.

Spergula arvensis $\mathrm{L}$.

Chenopodium album $\mathrm{L}$.

Raphanus raphanistrum $\mathrm{L}$.

Setaria viridis (L.) P. Beauv.

Echinochloa crus-galli (L.) P. B.

Capsella bursa-pastoris (L.) Med.

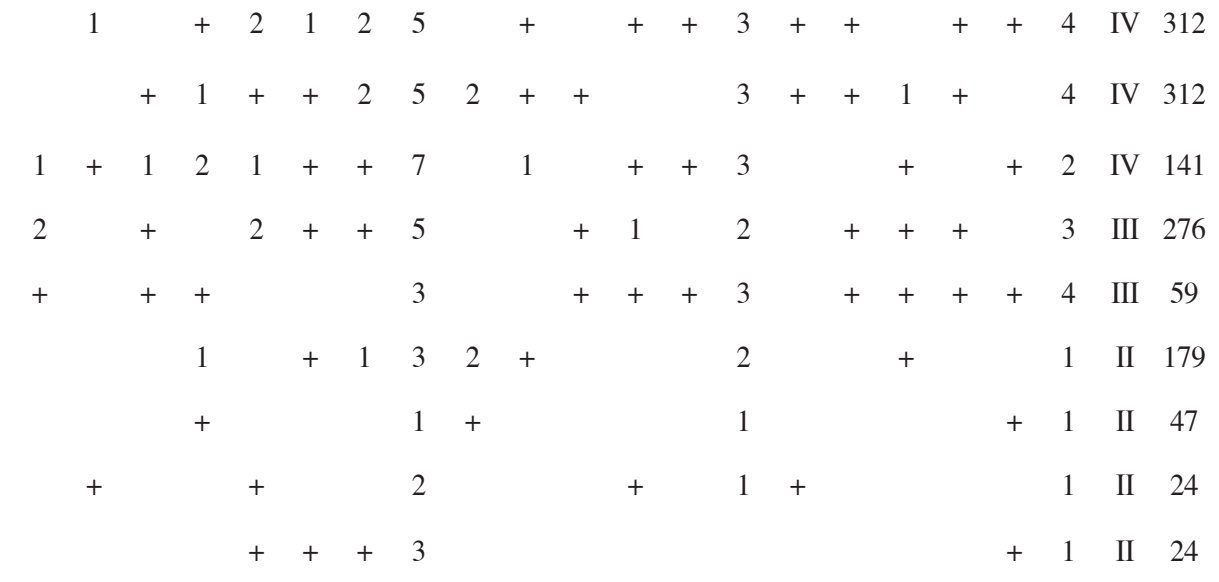

\section{Ch. Stellarietea mediae}

Viola arvensis Murr.

Fallopia convolvulus (L.) A. Löve

Conyza canadensis (L.) Cron.

Polygonum aviculare L.

Anthemis arvensis $\mathrm{L}$.

Centaurea cyanus $\mathrm{L}$.

Arnoseris minima (L.) Schweigg.

\& Körte

Myosotis arvensis (L.) Hill.
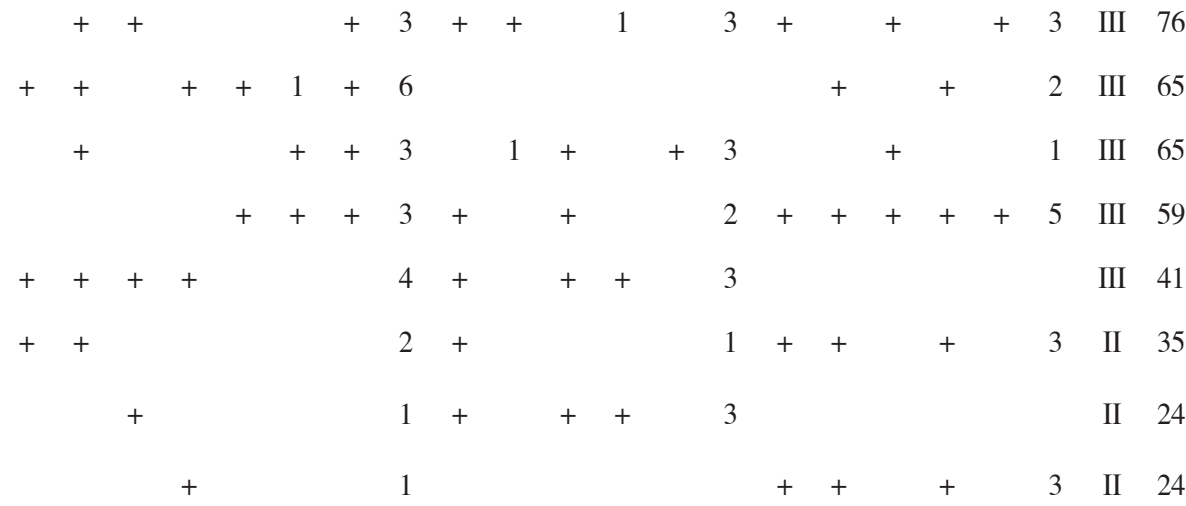

VI. Companions

Achillea millefolium L.

Convolvulus arvensis $\mathrm{L}$.

Erodium cicutarium (L.) L'hér.

Veronica arvensis L.

Polygonum lapathifolium L. subsp. lapathifolia

Gnaphalium sylvaticum L.

Elymus repens (L.) Gould

Agrostis stolonifera L.

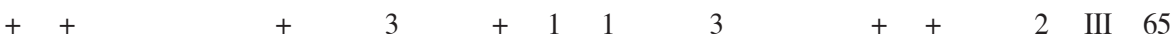

$$
\begin{aligned}
& +++++++7+2 \text { III } 53 \\
& +\quad+\quad+4+2+\quad+\quad+3 \text { III } 53 \\
& +++++5+\quad+\quad 2 \text { III } 41 \\
& \begin{array}{llllll}
2 & 2 & + & + & \text { II } & 144
\end{array} \\
& +\quad+114 \text { II } 71 \\
& \begin{array}{llllllll}
+ & 2 & + & + & &
\end{array}
\end{aligned}
$$

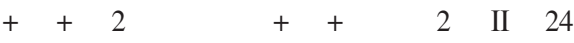

Sporadic species: II - Datura stramonium L. 8(r), 10(+), 12(+); Torylis japonica (Houtt.) DC. 10(+), 11(+); Descurainia sophia (L.) Weeb 11(+), 12(+); III - Stachys palustris L. 13(+), 14(+), 15(+); Bidens frondosa L. 14(+), 16(1); Polygonym amphibium L. 17(2); Potentilla anserina Borkh. 10(+); IV - Polygonum lapathifolium L. subsp. pallidum 5(+), 17(+); Geranium pusillum Burm. f. ex L. 14(+), 16(+); V Anthoxanthum aristatum Boiss. 2(+), 4(+), 10 (+); Stellaria media (L.) Vill. 4(+),6(+); Vicia hirsuta (L.) S.F. Gray 1(+); Matricaria maritima subsp. inodora (L.) Dostal 4(+); VI - Taraxacum officinale Wigg. 2(+), 8(+), (17); Galeopsis ladanum L. 1(+), 3(+); Plantago lanceolata L. 1(+), 17(+); Holcus mollis L. 3(+), 17(+); Poa annua L. 14(+), 16(+); Epilobium roseum Schreb. 14(+), 16(+); Equisetum arvense L. 14(+), 16(+); Gypsophila muralis L. 14(+), 16(+); Cerastium holosteoides Fr. em Hyl. 1(+); Cirsium arvense (L.) Scop. 1(+); Leontodon autumnalis L. 3(+); Holcus lanatus L. 9(+); Daucus carota L. 9(+); Veronica dillenii Cr. 10(+); Lotus corniculatus L. 12(+); Chamomilla suaveolens (Pursh) Rydb. 13(+); Erophila verna (L.) Chevall. 14(+).

Comments: numbers after species inform about the relevé in the table. S - phytosociological constancy, D - coverage index, zn -withered top leaves 


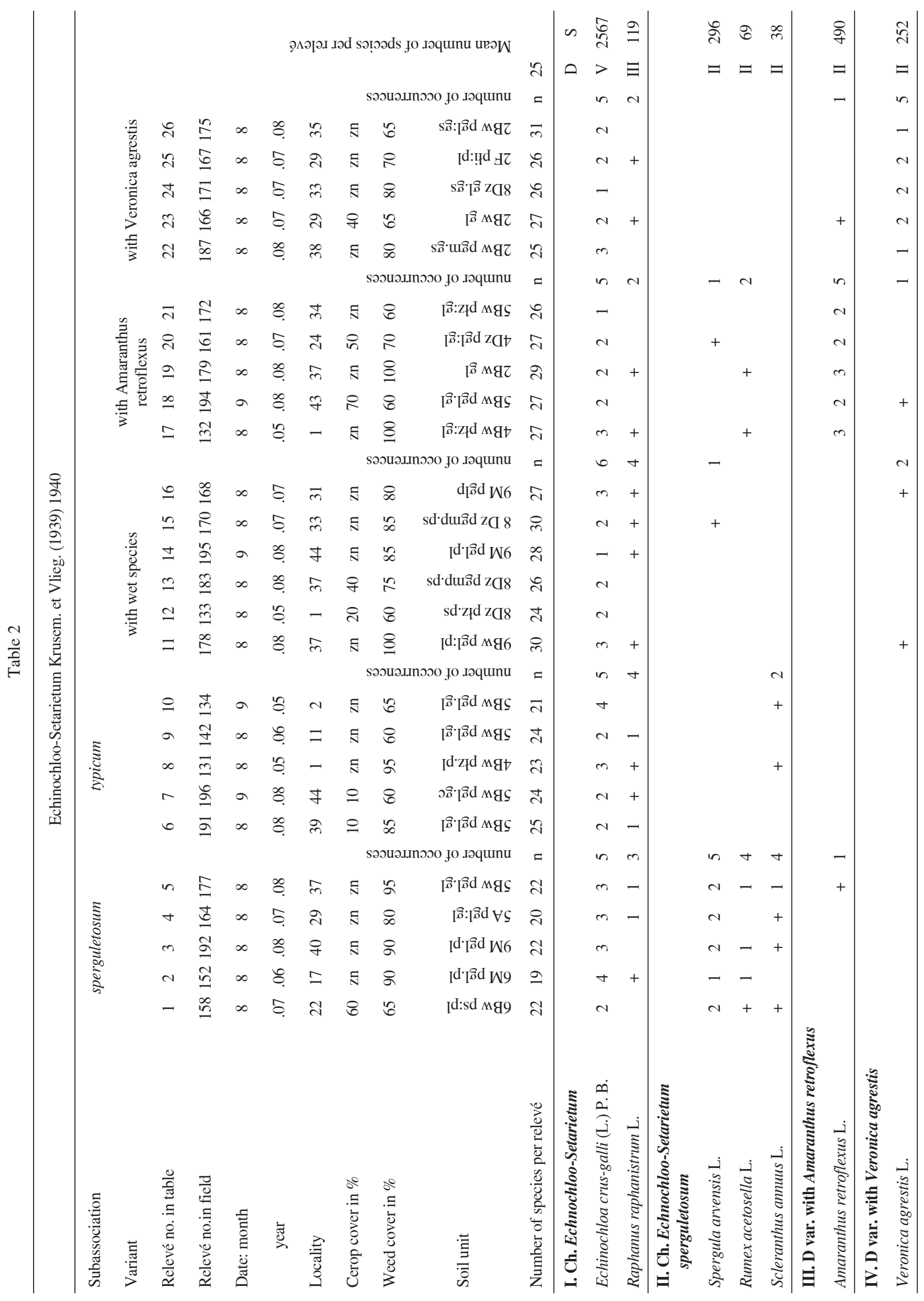




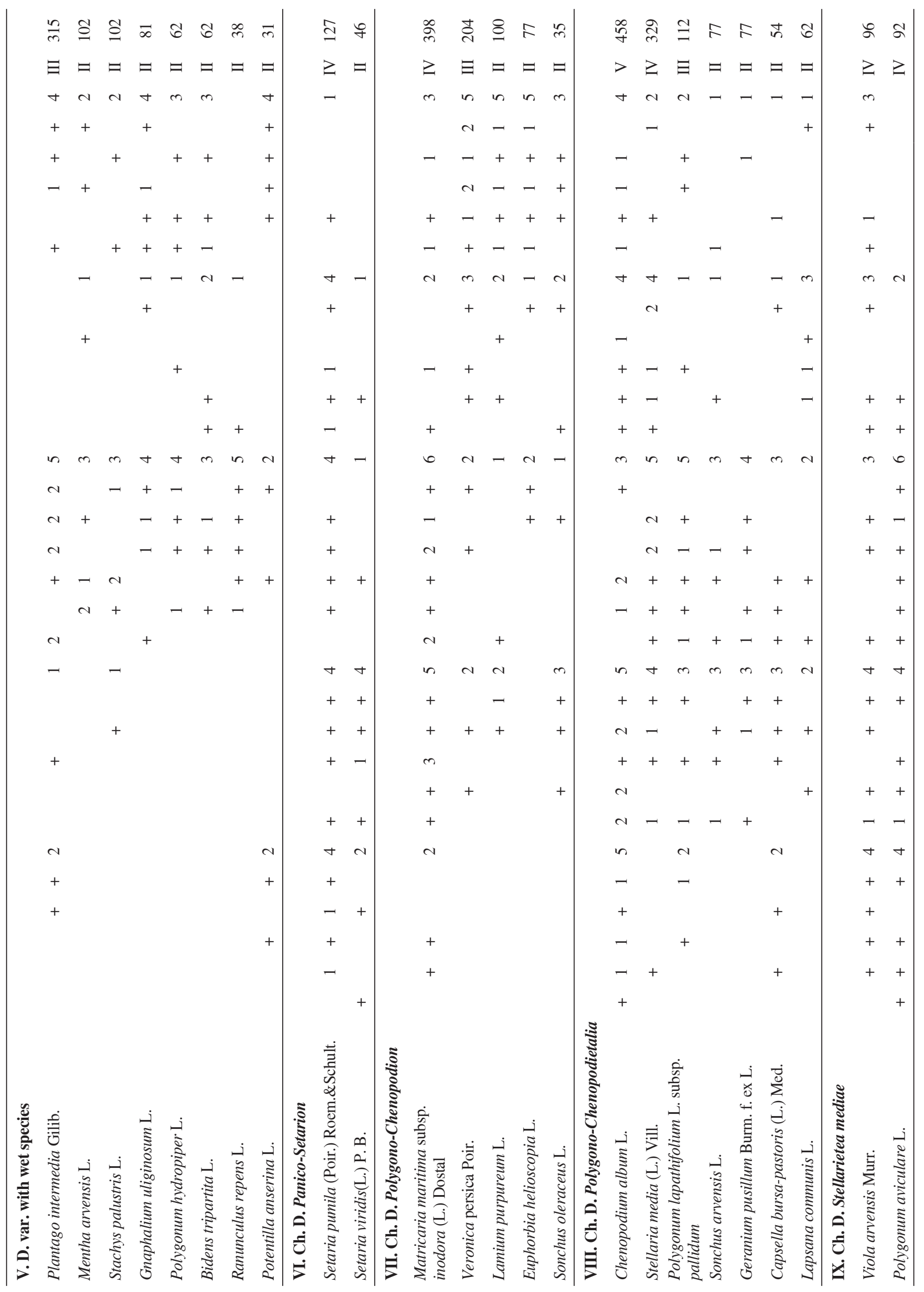




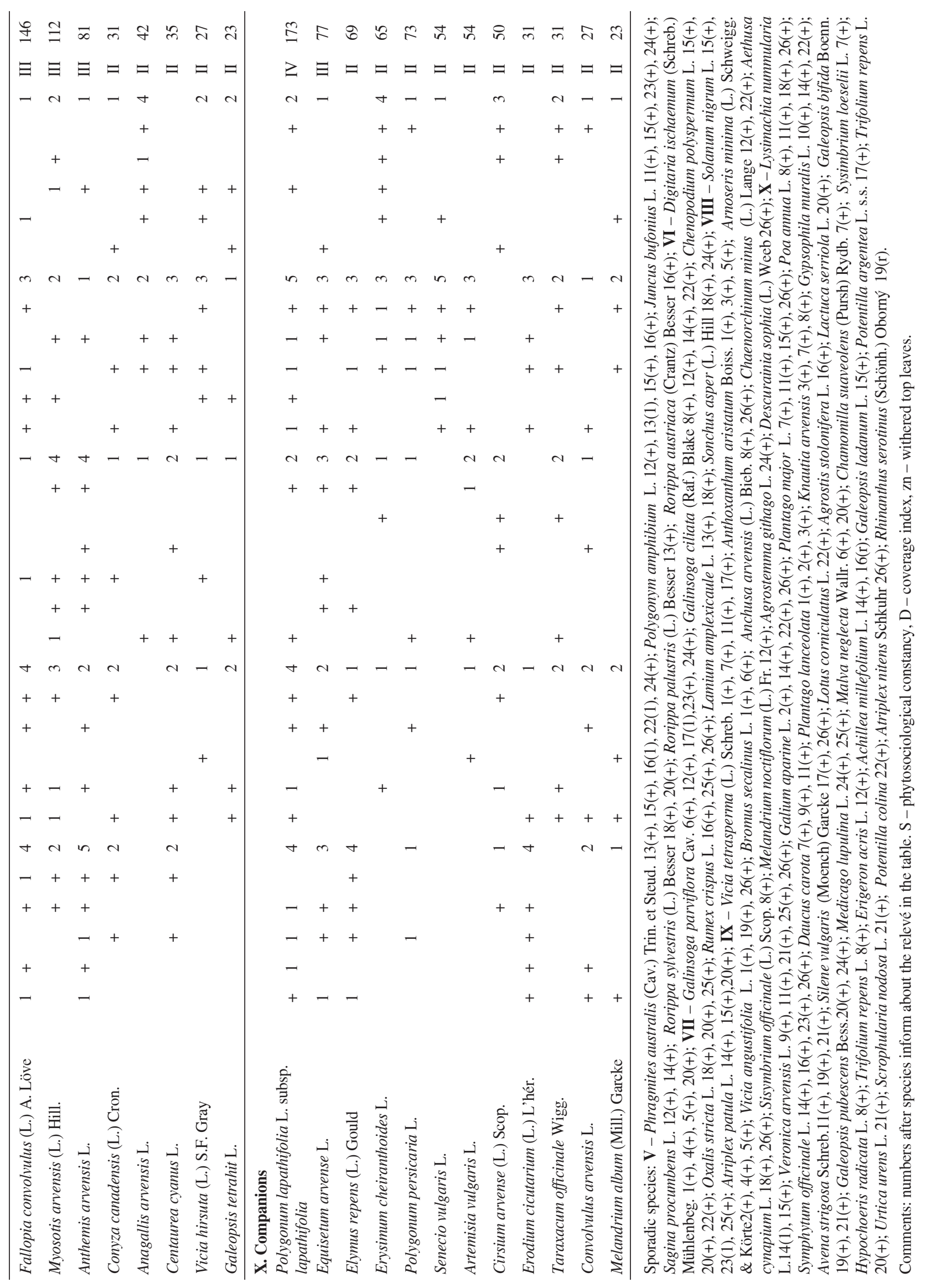


Table 3

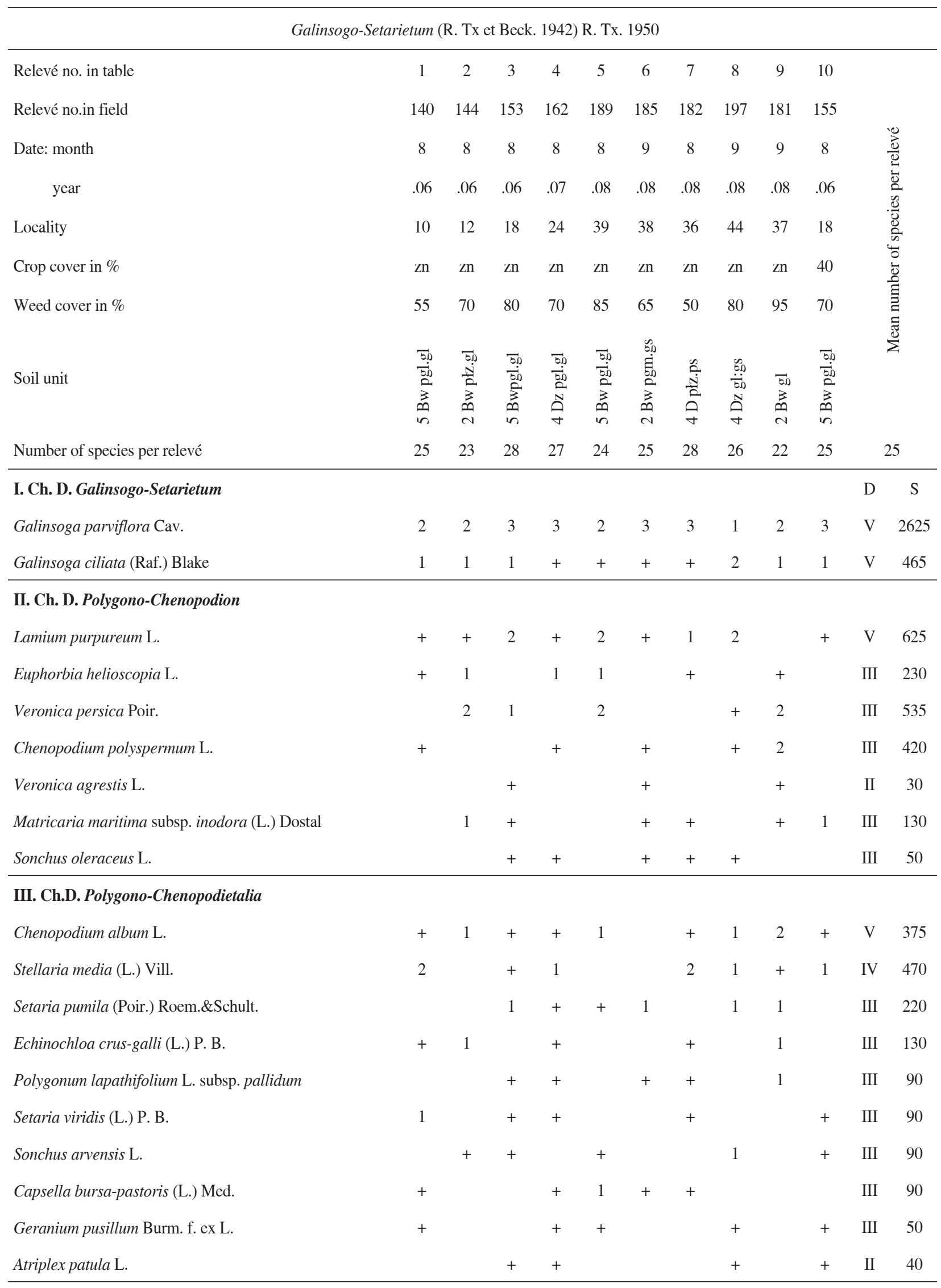




\section{Ch. D. Stellarietea mediae}

Viola arvensis Murr.

Anthemis arvensis $\mathrm{L}$.

Polygonum aviculare L.

Vicia hirsuta (L.) S.F. Gray

Fallopia convolvulus (L.) A. Löve

Myosotis arvensis (L.) Hill.

Conyza canadensis (L.) Cron.

Vicia angustifolia $\mathrm{L}$.

Centaurea cyanus L.

Apera spica-venti (L.) P. Beauv.

Anagallis arvensis $\mathrm{L}$.

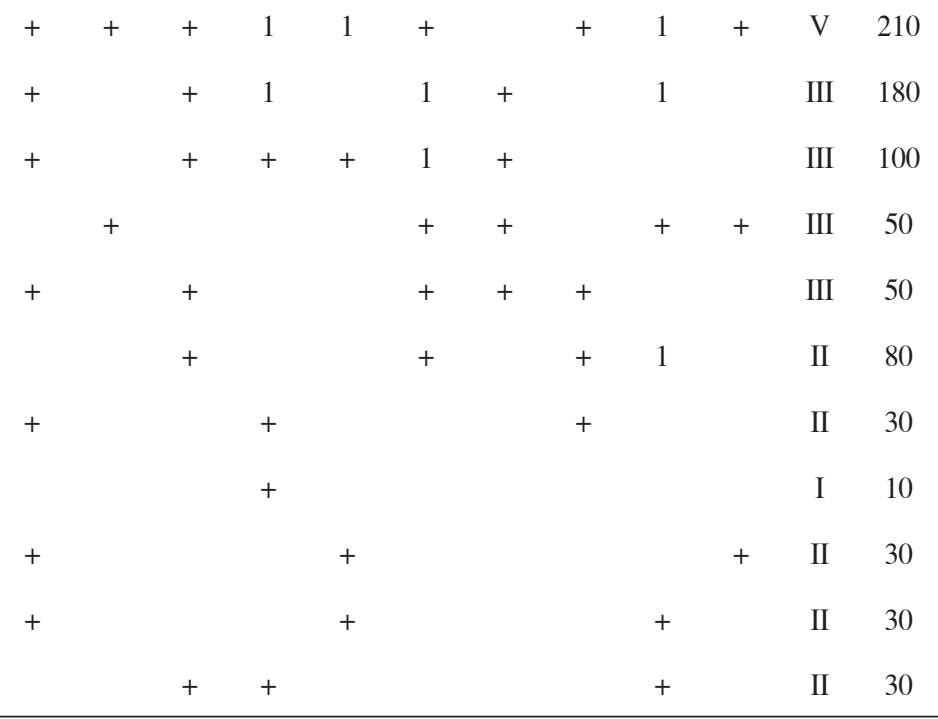

\section{Companions}

Erodium cicutarium (L.) L’hér.

Achillea millefolium $\mathrm{L}$.

Galium aparine L.

Veronica arvensis $\mathrm{L}$.

Erysimum cheiranthoides L.

Polygonum lapathifolium L. subsp. lapathifolia

Convolvulus arvensis L.

Galeopsis pubescens Bess.
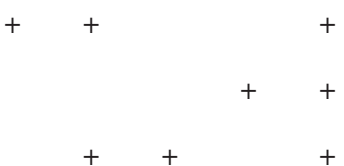

$+$

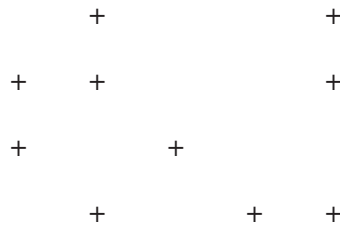

$+$

$+$ $+\quad+\quad$ III 50

$+\quad+\quad+$ III 50

III 50

II 70

$1 \quad$ II $\quad 70$

II 30

II 30

II $\quad 30$

Taraxacum officinale Wigg.

Artemisia vulgaris L.

$\begin{array}{rlllll} & + & + & \text { II } & 30 \\ + & + & & \text { II } & 30\end{array}$

Sporadic species: I - Lapsana communis L. 5(+); II - Sonchus asper (L.) Hill 1(+), 7(+); Oxalis stricta L. 6(+), 8(+); Rumex crispus L. 10(+); III - Raphanus raphanistrum L. 1(+), 6(+); Solanum nigrum L. 2(+); IV - Vicia villosa Roth. 3(+), 7(+); Descurainia sophia (L.) Weeb 3(+), 7(+); Aethusa cynapium L. 7(+), 10(+); Lactuca serriola L. 2(+); Vicia tetrasperma (L.) Schreb. 6(+); Anchusa arvensis (L.) Bieb. 7(+); Rumex acetosa L. 7(+); V - Elymus repens (L.) Gould 3(1), 4(1); Cirsium arvense (L.) Scop. 3(1), 5(+); Equisetum arvense L. 1(+), 8(1); Scrophularia nodosa L. 2(+), 8(+); Potentilla anserina L. 2(+), 7(+); Polygonum hydropiper L. 2(+), 9(+); Epilobium roseum Schreb. 3(+), 6(+); Gypsophila muralis L. 3(+), 10(+); Amaranthus retroflexus L. 6(+), 10(+); Melandrium album (Mill.) Garcke 7(+), 10(+); Agrostis stolonifera 8(+), 10(+); Polygonum persicaria L. 9(+), 10(1); Gnaphalium uliginosum L. 2(+); Medicago lupulina L. 4(+); Ranunculus repens L. 4(+); Prunella vulgaris L. 5(+); Veronica serpyllifolia L. 6(+); Leontodon autumnalis L. 6(+); Plantago major L. 7(+); Alopecurus geniculatus L. 8(+); Mentha arvensis L. 8(+); Ranunculus flammula L. 10(+); Rhinanthus serotinus (Schönh.) Oborný 4(r).

Comments: numbers after species inform about the relevé in the table. S - phytosociological constancy, D - coverage index, zn - withered top leaves 
Table 4

Lamio-Veronicetum politae Kornaś 1950

Relevé no. in table

Relevé no.in field

Date: month

$$
\text { year }
$$

Locality

Crop cover in \%

Weed cover in \%

Soil unit

\section{Lamio-Veronicetum politae Kornas 1950}

\begin{tabular}{|c|c|c|c|c|c|c|c|c|c|c|c|c|}
\hline 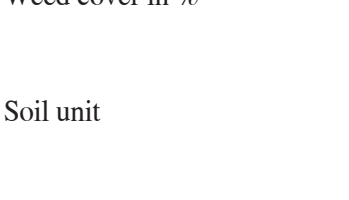 & $\begin{array}{l}\overline{00} \\
3 \\
\text { ते }\end{array}$ & 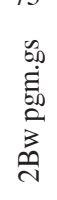 & 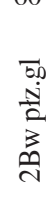 & $\begin{array}{l}50 \\
3 \\
\vec{n}\end{array}$ & 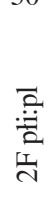 & 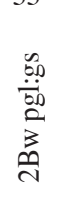 & 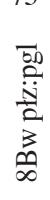 & 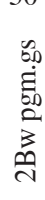 & 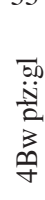 & $\begin{array}{l}\text { Do } \\
\text { 3 } \\
\text { ते }\end{array}$ & & \\
\hline Number of species per relevé & 31 & 28 & 27 & 26 & 25 & 24 & 24 & 27 & 25 & 26 & \multicolumn{2}{|c|}{26} \\
\hline I. Ch. Lamio-Veronicetum & & & & & & & & & & & $\mathrm{D}$ & $\mathrm{S}$ \\
\hline Lamium amplexicaule $\mathrm{L}$. & 2 & 1 & + & 1 & 1 & 1 & 1 & + & 1 & 1 & $\mathrm{~V}$ & 545 \\
\hline Veronica agrestis L. & 1 & 1 & & 1 & + & & + & & + & 2 & IV & 355 \\
\hline Veronica opaca Fr. & & & 1 & + & & & & 2 & + & & II & 245 \\
\hline Veronica polita Fr. & & & & + & & 1 & + & & + & & II & 80 \\
\hline
\end{tabular}

\section{Ch. Polygono-Chenopodion}

Veronica persica Poir.

Lamium purpureum $\mathrm{L}$.

Chenopodium polyspermum L.

Euphorbia helioscopia L.

Oxalis fontana Bunge

Sonchus asper (L.) Hill

Matricaria maritima subsp. inodora (L.) Dostal

Sonchus oleraceus L.

Galinsoga parviflora Cav.

Galinsoga ciliata (Raf.) Blake

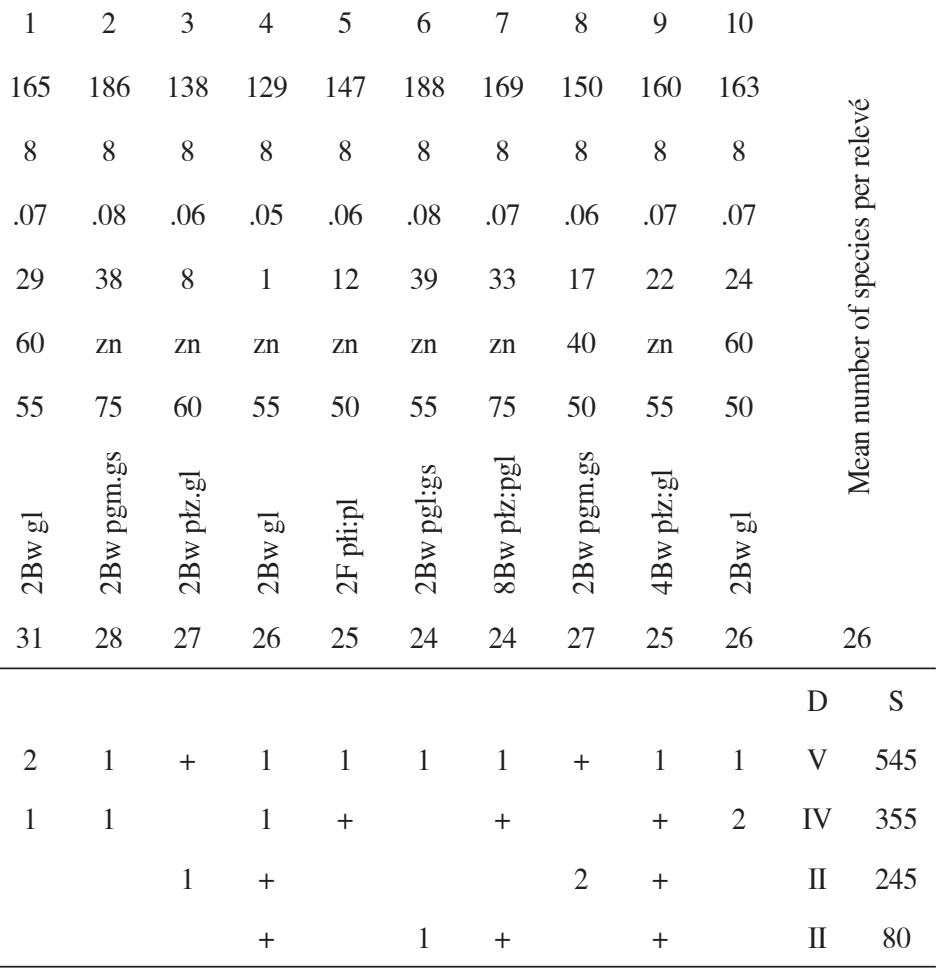

Lapsana communis L.

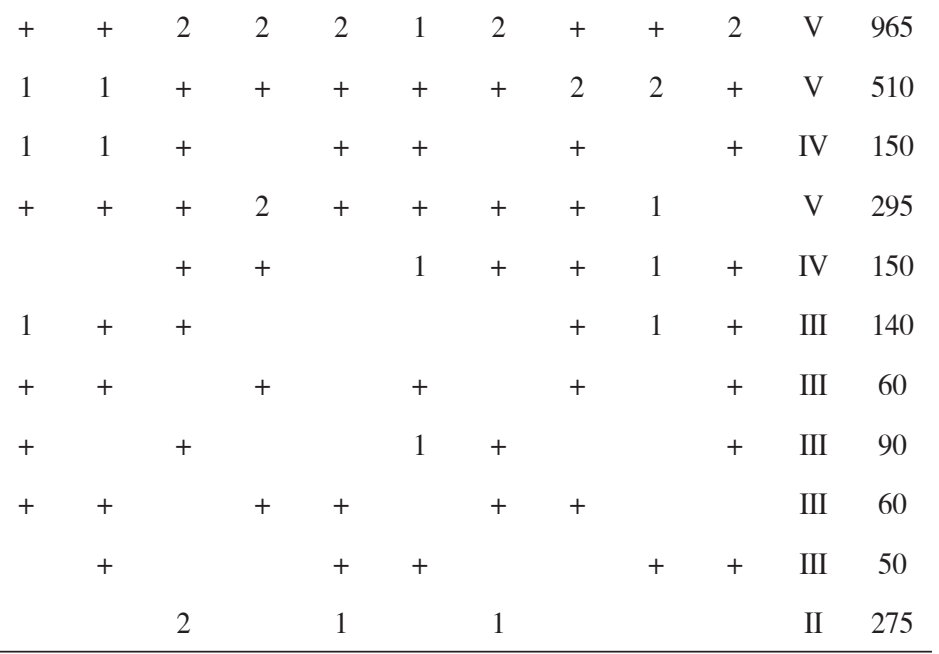

\section{Ch.D. Polygono-Chenopodietalia}

Chenopodium album $\mathrm{L}$.

Stellaria media (L.) Vill.

Echinochloa crus-galli (L.) P. B.

Polygonum lapathifolium L. subsp. pallidum

Sonchus arvensis L.

Capsella bursa-pastoris (L.) Med.

\begin{tabular}{|c|c|c|c|c|c|c|c|c|c|c|c|}
\hline 1 & + & + & + & + & + & + & + & + & + & $\mathrm{V}$ & 140 \\
\hline \multirow[t]{3}{*}{+} & + & 1 & + & + & 1 & 2 & + & 1 & + & V & 385 \\
\hline & & + & + & + & + & + & + & + & + & IV & 80 \\
\hline & & + & + & + & + & + & + & + & & IV & 70 \\
\hline \multirow[t]{2}{*}{+} & 3 & & + & + & + & & & + & + & III & 435 \\
\hline & + & & & & & + & + & + & + & III & 50 \\
\hline+ & & & & 1 & & 2 & + & + & & II & 255 \\
\hline
\end{tabular}

Solanum nigrum $\mathrm{L}$. 


\section{Ch. D. Stellarietea mediae}

Anagallis arvensis L.

Chaenorchinum minus (L.) Lange

Anchusa arvensis (L.) Bieb.

Aethusa cynapium L.

Myosotis arvensis (L.) Hill.

Anthemis arvensis $\mathrm{L}$.

Fallopia convolvulus (L.) A. Löve

\begin{tabular}{|c|c|c|c|c|c|c|c|c|c|c|c|}
\hline+ & + & & + & + & 2 & + & + & 1 & + & V & 295 \\
\hline+ & 1 & & + & 1 & + & + & + & + & + & $\mathrm{V}$ & 170 \\
\hline \multirow{5}{*}{+} & + & + & + & & + & & + & + & + & IV & 70 \\
\hline & + & & + & + & & & & & + & III & 50 \\
\hline & & + & + & + & & & + & + & & III & 50 \\
\hline & & & + & & & + & + & + & + & III & 50 \\
\hline & & + & & & & & + & & + & III & 30 \\
\hline
\end{tabular}

\section{Companions}

Ranunculus repens $\mathrm{L}$.

Plantago major L.

Elymus repens (L.) Gould

Cirsium arvense (L.) Scop.

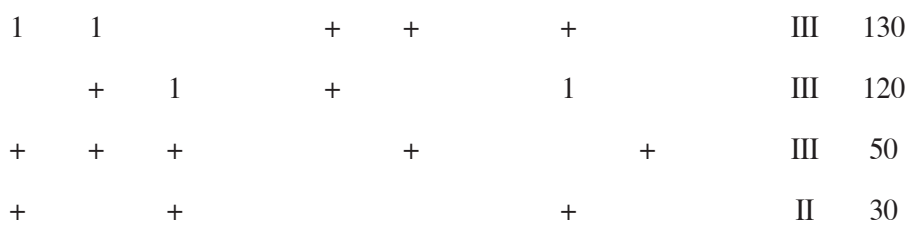

Sporadic species: II - Melandrium noctiflorum (L.) Fr. 1(+), 2(+); III - Setaria viridis (L.) P. B. 4(+), 6(+); Atriplex patula 1(+); Geranium pusillum Burm. f. ex L. 7(+); Setaria pumila (Poir.) Roem.\&Schult. 1(+); IV - Vicia angustifolia L. 1(+), 2(+); Polygonum aviculare L. 3(+), 7(+); Euphorbia exigua L. 1(+); Galeopsis tetrahit L. 2(+); Consolida regalis Gray 3(+); Thlaspi arvense L. 3(+); Descurainia sophia (L.) Weeb 5(+); Papaver rhoeas L. 5(+); Viola arvensis Murr. 7(+); Sisymbrium officinale (L.) Scop. 7(+); Agrostemma githago L. 8(+); Lactuca serriola L. 9(+); Centaurea cyanus L. 10 (+); V - Equisetum arvense L. 1(+); Polygonum lapathifolium L. subsp. lapathifolia 1(+); Trifolium medium L. 1(+); Polygonym amphibium L. 1(+); Symphytum officinale L. 1(+); Galium aparine L. 1(r); Epilobium roseum Schreb. 2(+); Convolvulus arvensis L. 2(+); Artemisia vulgaris L. 2(+); Stachys palustris L. 2(+); Melandrium album (Mill.) Garcke 3(+); Bidens tripartita L. 3(+); Ranunculus repens L. 3(+); Urtica urens L. 3(r); Veronica arvensis L. 4(+); Tanacetum vulgare L. 4(+); Polygonum hydropiper L. 4(+); Mentha arvensis L. 4(+); Gnaphalium uliginosum L. 5(+); Rorippa sylvestris (L.) Besser 5(+); Amaranthus retroflexus L. 6(+); Gypsophila muralis L. 6(+); Rorippa palustris (L.) Besser 6(+); Bidens frondosa L. 7(+); Phragmites australis (Cav.) Trin. et Steud. 8(+); Erysimum cheiranthoides L. 9(+); Juncus bufonius L. 10(+); Hieracium pilosella L. 10(+); Sagina procumbens L. 10(+); Medicago lupulina L. 10(+).

Comments: numbers after species inform about the relevé in the table. S - phytosociological constancy, D - coverage index, zn - withered top leaves.

\section{DISCUSSION}

Intensification of modern agriculture leads to impoverishment of agroecosystems. Many weed species disappear irreversibly, while others spread at a high rate and change the composition and structure of communities. Many authors focus on biodiversity preservation in agriculturally utilised areas and suggest various forms of species conservation, thereby conserving shrinking segetal communities (Ratyńska and Boratyn ski 2000; D o st at n y, 2004; S i c iń s k i and S i e r a d z k i, 2010). Such a role might also be fulfilled by landscape parks where the traditional farming system is maintained.

Soils of the Masovian Landscape Park have varied trophic and moisture conditions. Small areas under potato adjacent to fields cropped to cereals, meadows or ruderal communities additionally influence the diversity of segetal communities. These factors have contributed considerably to the high diversity within and inside the associations. Although no intermediate or impoverished associations or alliances were found in the area of the Masovian Landscape Park, reports by other authors examining various areas in different parts of Poland, Wnuk (1976), S zotkowski (1981), S zmeja (1989), A ni oł-Kwiatkowska (1990), Kozak (2002), S iciński (2003), Węgrzynek (2005), Skrajna and Skrzyczyńska (2008), Rzymowska and Skrzyc zyńska (2007), have shown that the floristic distinctiveness of agrophytocenoses is diminishing.

4 associations were found in fields cropped to potato in the study area, that is, Digitarietum ischaemi, Echinochloo-Setarietum, Galinsogo-Setarietum and Lamio-Veronicetum politae, the first two associations being internally diverse. Due to its Sub-Atlantic character, Digitarietum ischaemum reaches the south-eastern boundary of its occurrence in Poland. According to many authors (Aniol-Kwiatkowska, 1990; K o z a k, 2002; W ę g r z y n e k, 2005), the association is disappearing from agrophytocenoses as the poorest habitats are excluded from cultivation, the production potential of these habitats increases, and they are allotted for construction or afforestation. 
What is interesting is the presence of phytocenoses with ruderal species in parts of the patches, which can result from small cropped areas and the proximity of buildings and fallow land. It is also indicative of partial ruderalisation of these communities. The process of infiltration of ruderal species into segetal communities in Poland has been reported by $\mathrm{K}$ a p e luszny (2000), Misiewicz et al. (2000), Kut y na et al. (2010), and Trąb a (2010).

Some Digitarietum ischaemi patches included a group of wet shallow-rooting species. Wet species are quite rare in this association. Similar phytocenoses with wet species in fields cropped to potato have been reported by Głazek and Kowalik (1983), Szmeja (1994), Skrajna and Skrzyczyńska (2008), Rzy mow ska and Skrzyczyńska (2007), and S i c ińs k i (2003).

Well-developed patches of Lamio-Veronicetum politae with a full range of the association's diagnostic species established on fertile nutrient-rich soils. The analysed association found in some areas of Poland is usually mentioned in its impoverished form with a rare occurrence of Veronica sp. (K or c z y ński, 1998; S z meja, 1989; Kozak, 2002; W ę grzynek, 2006). According to Wnuk (1987) and Aniol- Kw i at kowska (1990), Veronica polita, Veronica opaca and Veronica agrestis are the species that best characterise Lamio-Veronicetum politae.

\section{CONCLUSIONS}

1. Potato fields in the Masovian Landscape Park cover small areas and are more and more difficult to find.

2. Plant communities established in the investigated crops are diversified and floristically rich, which reflects substantial soil variability and extensive farming.

3. The type of farming in such areas is particularly important in preserving the segetal flora diversity of agricultural landscape.

\section{Acknowledgements}

Research supported by the Ministry of Science and Higher Education of Poland as the part of statutory activities of Department of Agricultural Ecology, Siedlce University of Natural Sciences and Humanities.

\section{REFERENCES}

Anioł-Kwiatkowska J. 1990. Zbiorowiska segetalne Wału Trzebnickiego. Florystyczno-ekologiczne studium porównawcze. / Segetal communities of Wał Trzebnicki. Floristic and ecological comparative study. Wyd. Uniw. Wr. Pr. Bot.: 46-230. (in Polish)
Dostatny D.F. 2004. Preservation of weeds diversity in protected areas. Biul. Ogrodów Bot. 13: 79-83.

Głazek T., Kowalik B. 1983. Zbiorowiska chwastów polnych gminy Raków w województwie kieleckim. I Field weed communities of the Raków commune of the Kielce viovodeship. Studia Kieleckie, 2(38): 7-28.

Główny Urząd Statystyczny (GUS), 2011. www.stat.gov.pl

Kapeluszny J. 2000. Obserwacje z okolic Lublina nad występowaniem niektórych gatunków roślin ruderalnych w uprawach rolniczych i ogrodniczych. / Observations on the occurrence of some ruderal plants in agricultural and horticultural crops in the Lublin area. Ann. UMCS, Sec. E 55: 77-84. (in Polish)

Korczyński M. 1998. Przemiany i stan flory segetalnej Bydgoszczy. / Transformations and the state of segetal flora in Bydgoszcz. Acta Univ. Lodz., Folia Bot. 13: $65-72$.

Kozak M. 2002. Zbiorowiska segetalne gminy Rudniki (woj. Opolskie). / Segetal communities in the Rudniki municipality area (Opole Viovodship). Fragm. Flor. Geobot. Polonica, 9: 219-272. (in Polish)

Kutyna I., Młynkowiak E., Leśnik T.2010. Struktura fitosocjologiczna fitocenoz zbóż ozimych na tle warunków glebowych południowo-zachodniej Niziny Szczecińskiej i terenów do niej przyległych. / Phytosociological structure of phytocenosis in winter cereals on the background of soil conditions on the area of south-western part of Szczecińska Lowland and areas adjacent to this lowland. Fragm. Agronom. 27(3): 86-101. (in Polish)

Matuszkiewicz. W. 2007. Przewodnik do oznaczania zbiorowisk roślinnych Polski. / Guide for identification of Poland's plant communities. Vademecum geobotanicum Państwowe Wydawnictwo Naukowe, Warszawa, 537 pp. (in Polish)

Mirek Z., Piękoś-Mirkowa H., Zając A., Zając M. 2002. Flowering plants and peridophytes of Poland a checklist.- [In:] Z. Mirek (ed.) Biodiversity of Poland 1, s. 442. W. Szafer Institute of Botany, Polish Academy of Sciences, Kraków.

Misiewicz J., Łupacz L., Sawilska A.K., Stypczyńska Z. 2000. Zasoby flory ruderalnej jako źródło potencjalnych chwastów segetalnych na terenie gminy Osielsko. / Ruderal flora as a source of potential segetal weeds within the commune of Osielsko. Zesz. Nauk., Bydgoszcz, 226, ser. Rol. 45: 85-89. (in Polish)

Paw low ski B. 1972. Skład i budowa zbiorowisk roślinnych oraz metody ich badania. [In:] Szafer Wł., Zarzycki K.: Szata roślinna Polski. / The vegetation of Poland. T. I. PWN, Warszawa: 237-268. (in Polish)

Ratyńska H., B oratyński A. 2000. Czynna ochrona roślin i zbiorowisk segetalnych i ruderalnych. / Acive protection of segetal and ruderal plants and vegetation. Przegląd Przyr., XI, 2-3: 43-56. (in Polish)

Rzymowska Z., Skrzyczyńska J. 2007. Plant communities of cultivated fields of the Podlaski Przełom Bugu Mesoregion. Acta Agrobot. 60 (1): 191-205. 
Siciński J.T. 2003. Agrofitocenozy dorzecza środkowej Warty i Bzury - stan, dynamika i zagrożenia. / Agrophytocenoses of the middle Warta and Bzura river systems - present state, dynamics and threats. Wyd. UŁ. Rozp.: 1-69. (in Polish).

Siciński J.T., Sieradzki J. 2010. Protection of segetal flora and vegetation in Poland (historical outline). Plant Breding and Seed Science, 61: 123-131.

Skrajna T., Skrzyczyńska J. 2008. Plant communities and associations of root crops of the Kałuszyńska Upland. Acta Agrobot. 61 (2): 239-249.

Skrajna T., Skrzyczyńska J., Ługowska M. 2009. Segetal communities of cereal cultivations of the Mazowiecki Landscape Park. Acta Agrobot. 62 (1): 171-186.

Skrajna T., Ługowska M. 2010. Stubble field plant communities of the Mazowiecki Landscape Park. Acta Agrobot. 63 (2): 189-205.

Szmeja K. 1989. Roślinność pól uprawnych Wzniesień Elbląskich. / Crop field vegetation of Elbląskie Hills. Tow. Przyj. Nauk, Acta Biol., 7: 1-66.

Szmeja K. 1994. Roślinność pól uprawnych Zaborskiego Parku Krajobrazowego. / Farmland vegetation of the Zaborski Landscape Park. Fragm. Flor. Geobot., Ser. Polonica, 1: 157-180. (in Polish)

Szotkowski P. 1981. Chwasty upraw okopowych i zbóż ozimych w południowo-wschodnim obszarze Śląska Opolskiego. / Weeds of root crops and winter cereals in the south-eastern area of Opole Silesia. Opolskie Tow. Przyj. Nauk., Warszawa-Wrocław, PWN: 3-190. (in Polish)

Trąba Cz. 2010. Gatunki migrujące na pola uprawne na rędzinach z ekosystemów występujących w sąsiedztwie. / Species migrating into croplands on rendzinas from neighbouring ecosystems. Fragm. Agronom. 27 (2): 156-163. (in Polish)

Węgrzynek B. 2005. Roślinność segetalna Wyżyny Śląskiej. Cz. IV. Zbiorowiska chwastów upraw okopowych ze związku Panico-Setarion Siss. 1946. / The segetal vegetation of the Silesian Upland. Part IV. Root crop weed communities of the Panico-Setarion Siss. 1946 Alliance. Weed communities of maize crop Natura Silesiae Superioris, Centrum Dziedzictwa Przyrody Górnego Śląska, 8: 39-53. (in Polish)

Węgrzynek B. 2006. Roślinność segetalna Wyżyny Śląskiej. Cz. V. Zbiorowiska chwastów upraw okopowych ze związku Eu-Polygono-Chenopodiom polyspermi
(Koch 1926) Siss. 1946. / Segetal vegetation of the Silesian Upland. Part V. Root crop weed communities of the Eu-Polygono-Chenopodion polyspermi (Koch 1946) Siss. 1946 Alliance. Weed communities of maize crop. Natura Silesiae Superioris, Centrum Dziedzictwa Przyrody Górnego Śląska, 9(2005): 63-83. (in Polish)

Wnuk Z. 1976. Zbiorowiska chwastów segetalnych Pasma Przedborsko-Małogoskiego i terenów przyległych. Cz. I. Zbiorowiska upraw okopowych. / Segetal weed communities of the Przedborsko-Małogoskie Hills Range and adjacent areas. Part 1. Root crop communities. Acta Univ. Lodz., Zesz. Nauk. UŁ, ser. II, 14: 85-122. (in Polish)

Wnuk Z. 1987. Zespół Lamio-Veronicetum politae Kornaś 1950 w Polsce. / The Lamio-Veronicetum politae Kornaś 1950 association in Poland. Zesz. Nauk. AR w Krakowie, 216 (19): 95-136. (in Polish)

\section{Zbiorowiska chwastów upraw ziemniaka (Solanum tuberosum L.) Mazowieckiego Parku Krajobrazowego}

\section{Streszczenie}

Praca przedstawia charakterystykę zbiorowisk segetalnych wykształcających się w uprawach ziemniaka na terenie Mazowieckiego Parku Krajobrazowego. Do opracowania zbiorowisk wykorzystano 64 zdjęcia fitosocjologiczne wykonane w 45 miejscowościach. Na badanym terenie w uprawach ziemniaka stwierdzono występowanie 4 zespołów: Digitarietum ischaemi, Echinochloo-Setarietum, Galinsogo-Setarietum, Lamio-Veronicetum politae.

Najbardziej zróżnicowane pod względem florystycznym były fitocenozy zakwalifikowane do zespołów Digitarietum ischaemi i Echinochloo-Setarietum. $\mathrm{W}$ ramach tych asocjacji wyróżniono niższe jednostki syntaksonomiczne $\mathrm{w}$ randze podzespołów i wariantów. Fitocenozy te były często spotykane, występowały w szerokim spektrum siedliskowym zarówno pod względem troficznym, jak i wilgotnościowym. Na glebach żyznych rzadko i na niewielkich powierzchniach notowano płaty zespołu Lamio-Veronicetum politae. Charakteryzowały się one pełnym składem gatunków diagnostycznych dla tej asocjacji. 
